\title{
¿Cuándo dos exámenes seriados de laboratorio representan un cambio en el estado de salud de un paciente?
}

\author{
ANA MARÍA GUZMÁN D.
}

Departamento de Laboratorios Clínicos, Facultad de Medicina Pontificia Universidad Católica de Chile. Santiago de Chile.

Recibido el 7 de junio 2010, aceptado el 14 de junio de

Correspondencia a: Dra. Ana María Guzmán D. Departamento de Laboratorios Clínicos, Facultad de Medicina Pontificia Universidad Católica de Chile Santiago de Chile. E-mail: amguzman@med. puc.cl

\section{When two consecutive laboratory results indicate a change in health status in a patient?}

Sources of variation between two serial tests must be considered in interpreting if there was a clinically significant change. The main causes of variation are the biological variation coefficient (CVB) of the test in question, which must be obtained from the literature, and the analytical coefficient of variation (CVA) of the same test, which must be obtained from the internal quality control laboratory data. With both data we can calculate the critical difference or "reference change value" which helps us to decide whether there was a real change in the patient's health.

(Rev Med Chile 2010; 138: 780-783).

Key words: Biological markers; Clinical chemistry tests; Clinical laboratories.

\section{RESUMEN}

Antes de interpretar si los resultados de dos exámenes de laboratorio consecutivos representan un cambio clinicamente significativo, se deben considerar otras fuentes de variación de las mediciones, entre ellas el coeficiente de variación biológico (CVB) y analítico (CVA). El CVB es un parámetro fijo que se obtiene de la literatura médica y en páginas web y el CVA se calcula a partir de los datos del control de calidad interno del laboratorio en que se realiza el examen. Con el CVB y el CVA podemos calcular la diferencia crítica o "reference change value", lo cual contribuirá a decidir si hubo un cambio real en el estado de salud del paciente.

\section{DE LA VIDA REAL}

Usted es un médico general y recibe en su consulta un paciente de 40 años de edad, sano, sin antecedentes mórbidos de importancia. Trae un perfil bioquímico normal de hace 2 semanas atrás y un estudio de lípidos del día anterior. Llama la atención que aún cuando ambos exámenes fueron realizados en el mismo laboratorio, con el mismo método, el colesterol tuvo un cambio de $185 \mathrm{mg} / \mathrm{dL}$ a $220 \mathrm{mg} / \mathrm{dL}$ (valor de referencia menor a $200 \mathrm{mg} / \mathrm{dL}$ ).

¿Son discordantes estos resultados del nivel sérico de colesterol? 


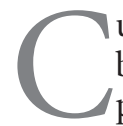
uando realizamos dos exámenes de laboratorio seriados para el control de un paciente, es muy importante que el médico conozca todos los elementos que pueden ser fuentes de variación en las mediciones. Excluyendo la falta de armonización de las técnicas utilizadas para las determinaciones, lo cual ya fue comentado en un artículo anterior de esta serie ${ }^{1}$, existen 4 fuentes principales de variación en un examen ${ }^{2}$ :

1. Variación pre-analítica.

2. Variación biológica.

3. Variación analítica.

4. Variaciones debidas a cambios en el estado de salud.

\section{Variación pre-analítica:}

Corresponden a las variaciones que ocurren antes de que la muestra entre a la etapa de análisis. Fuentes de variación de esta etapa son, por ejemplo: la hora de la toma de muestra, tiempo de aplicación del torniquete para la venopunción, tipo de anticoagulante usado, orden en la recolección de los tubos, temperatura a la cual las muestras fueron transportadas, tiempo y velocidad de centrifugación, tiempo que transcurrió entre la toma de muestra y el análisis, condiciones de congelamiento y descongelamiento (si fue necesario congelar las muestras), etc. Es importante que la variación pre-analítica sea minimizada al máximo a través de la estandarización de todos los procedimientos de toma de muestra, recolección, transporte y manipulación de ellas, antes de que ingresen al análisis mismo. Además de los procedimientos escritos que debe manejar el personal de enfermería, deben existir instructivos dirigidos a los pacientes ambulatorios, cuando se prevén variaciones debidas a ejercicio físico, dieta, etc. que el paciente pueda controlar ${ }^{3}$.

\section{Variación biológica:}

Varios compuestos presentes en los líquidos biológicos y que son medidos en los laboratorios clínicos (analitos) presentan ritmos biológicos, como, por ejemplo, el cortisol (ritmo diario), las hormonas femeninas luteínizante (LH) y folículo-estimulante (FSH) (ritmo mensual). Otra situación similar en que las concentraciones de un analito pueden variar en forma importante, es en la medición de niveles plasmáticos de drogas terapéuticas (antes y después de la administración de la dosis). En estos casos es importante realizar la toma de muestra tomando en consideración estas variaciones.

Sin embargo, la mayoría de los analitos no presentan variaciones significativas debidas a ritmos biológicos, pero sí una oscilación alrededor de un punto homeostático, la cual se considera fisiológica y se denomina variación biológica ${ }^{4}$. Para establecer la magnitud de esta variación, se estudian diariamente, durante aproximadamente 20 días, los parámetros de interés en un grupo de sujetos sanos, minimizando las variables preanalíticas. Con los datos se calcula el promedio $(\overline{\mathrm{x}})$ y la desviación estándar (DE), y se obtiene el Coeficiente de Variación Biológico (CVB) que se expresa como porcentaje.

$$
\mathrm{CVB}=\frac{\mathrm{DE}}{\overline{\mathrm{X}}} \times 100
$$

El CVB se denomina intraindividual, si se utilizan los datos seriados de un individuo o interindividual, si se ocupan los datos del total de individuos estudiados.

Múltiples estudios han determinado que esta variación es constante para cada analito; no cambia con la edad, el género, ni la etnia, y se ha visto además que es independiente del número de sujetos estudiados y del método analítico utilizado. Así, se han llegado a establecer listas con el CVB de la mayoría de los analitos de importancia clínica, las cuales están fácilmente disponibles en la literatura médica y en páginas web (Tabla 1$)^{4,5}$.

Sin embargo, recientemente se ha establecido que el CVB de algunos analitos varía en estados de enfermedad (Tabla 2$)^{6}$. Este aumento es especialmente relevante cuando se trata de la variación biológica de analitos cuya medición se utiliza para el seguimiento del paciente o en estudios seriados. Por ejemplo, la hemoglobina glicosilada, cuyos niveles se utilizan para controlar a los pacientes diabéticos, tiene un CVB de 1,9\% en individuos sanos, pero aumenta a $8,8 \%$ en individuos con diabetes mellitus tipo 1 y a $4,3 \%$ en pacientes con diabetes tipo 2. Lo mismo sucede con marcadores tumorales como el antígeno carcinoembrionario (CEA) que presenta un CVB de $13 \%$ en sujetos sanos y aumenta a 44,9\% en sujetos con cáncer de colon. Esta situación dificulta el control de los pacientes, ya que aumentos o disminuciones del valor del marcador tumoral, no siempre serán indicativos de empeoramiento o control de la enfermedad. 
Tabla 1. Coeficiente de variación biológico para algunos analitos de uso frecuente en medicina ${ }^{3}$

\begin{tabular}{|lcc|}
\hline Analito & $\begin{array}{c}\text { Coeficiente de variación } \\
\text { biológico (\%) }\end{array}$ \\
Sodio & $\begin{array}{c}\text { Intra- } \\
\text { individual }\end{array}$ & $\begin{array}{c}\text { Inter- } \\
\text { individual }\end{array}$ \\
\hline Cloro & 0,7 & 1,0 \\
\hline Calcio & 1,2 & 1,5 \\
\hline Proteínas & 1,9 & 2,8 \\
\hline Albúmina & 2,7 & 4,0 \\
\hline Potasio & 3,1 & 4,2 \\
\hline Glucosa & 4,8 & 5,6 \\
\hline Colesterol & 5,7 & 6,9 \\
\hline Colesterol HDL & 6,0 & 14,9 \\
\hline Colesterol LDL & 7,1 & 19,7 \\
\hline LDH & 8,3 & 25,7 \\
\hline Triglicéridos & 8,6 & 14,7 \\
\hline CK & 20,9 & 37,2 \\
\hline Bilirrubina total & 25,6 & 40,0 \\
\hline
\end{tabular}

\section{Variación analítica}

La variación analítica o imprecisión de un método cuantitativo corresponde a la dispersión de los valores (desviación estándar) obtenidos para una misma muestra alrededor del promedio $(\overline{\mathrm{X}})$, expresado en porcentaje, que se calcula a partir de la siguiente fórmula:

$$
\mathrm{CVA}=\frac{\mathrm{DE}}{\overline{\mathrm{x}}} \times 100
$$

Si un método presenta un CVA pequeño, quiere decir que si repetimos la determinación en una misma muestra n veces, la medición tendrá una alta reproducibilidad o fiabilidad, ambos términos utilizados frecuentemente en la literatura médica ${ }^{7}$.

En el laboratorio clínico, para todas las técnicas cuantitativas se utilizan materiales controles, que son generalmente comerciales de matriz similar a suero, orina $\mathrm{u}$ otros fluidos corporales. Estos controles debe ser medidos en dos concentraciones diferentes al menos una vez al día, para poder asegurar que las técnicas estén funcionando ade-
Tabla 2. Coeficiente de variación biológico de algunos marcadores tumorales y hemoglobina glicosilada en individuos sanos y enfermos ${ }^{5}$

\begin{tabular}{|lccl|}
\hline Analito & \multicolumn{2}{c|}{$\begin{array}{c}\text { Coeficiente de variación } \\
\text { biológico (\%) }\end{array}$} \\
\hline Individuos & $\begin{array}{c}\text { Individuos } \\
\text { sanos }\end{array}$ & $\begin{array}{c}\text { enfermos } \\
\text { (Cirrosis) }\end{array}$ \\
\hline CA-125 & 12 & 40 (Cáncer ovárico) \\
\hline CA 15,3 & 29 & $46 \quad$ (Cáncer de \\
\hline CEA & 6,2 & 16,7 mama) \\
\hline $\begin{array}{l}\text { Hemoglobina } \\
\text { glicosilada }\end{array}$ & 13 & 44,9 (Cáncer de colon) \\
& 1,9 & 4,3 (Diabetes mellitus \\
tipo 2) \\
8,8 (Diabetes mellitus \\
tipo1)
\end{tabular}

cuadamente. La repetición diaria de la medición de estos controles de concentración conocida, entregan en cada laboratorio los datos para calcular la imprecisión de las técnicas, e incluso hoy en día existen "software" capaces de manejar el control de calidad y entregar los datos de imprecisión cada vez que se ingresan nuevos valores de control.

\section{Variación debida a un cambio en el estado de salud del paciente}

Para establecer si un cambio de valores en dos exámenes seriados representa un cambio en el estado de salud, deben considerarse todas las fuentes de variación nombradas. La variación total de un test de laboratorio incluye, entonces, la variación pre-analítica, la variación biológica intraindividual y la variación analítica del test y se expresa de la siguiente forma ${ }^{3,8}$ :

$$
\mathrm{CV}_{\mathrm{T}}^{2}=\mathrm{CV}_{\mathrm{P}}^{2}+\mathrm{CV}_{\mathrm{B}}^{2}+\mathrm{CV}_{\mathrm{A}}^{2}
$$

Donde:

$\mathrm{CV}_{\mathrm{T}}=$ coeficiente de variación total

$\mathrm{CV}_{\mathrm{B}}^{\mathrm{T}}=$ coeficiente de variación biológico intraindividual

$\mathrm{CV}_{\mathrm{A}}=$ coeficiente de variación analítico

Si minimizamos las variables pre-analíticas la variación total queda como:

$$
\mathrm{CV}_{\mathrm{T}}{ }^{2}=\mathrm{CV}_{\mathrm{B}}^{2}+\mathrm{CV}_{\mathrm{A}}^{2}
$$


En dos exámenes consecutivos o seriados debemos calcular la diferencia crítica o "reference change value" (RCV) que corresponde a:

$$
\begin{aligned}
& \mathrm{RCV}=\mathrm{Zx}\left(\left(\mathrm{CV}_{\mathrm{B}}{ }^{2}+\mathrm{CV}_{\mathrm{A}}{ }^{2}\right)+\left(\mathrm{CV}_{\mathrm{B}}{ }^{2}+\mathrm{CV}_{\mathrm{A}}{ }^{2}\right)\right)^{1 / 2} \\
& \mathrm{RCV}=2^{1 / 2} \times \mathrm{Z} \times\left(\mathrm{CV}_{\mathrm{B}}{ }^{2}+\mathrm{CV}_{\mathrm{A}}{ }^{2}\right)^{1 / 2}
\end{aligned}
$$

Considerando un score $\mathrm{Z}$ de 1,96 para cambios significativos con probabilidad del $95 \%$ y de 2,58 para cambios muy significativos con probabilidad del 99\%.

En el ejemplo de la vida real, tenemos un cambio en el valor del colesterol medido de $185 \mathrm{mg} /$ $\mathrm{dL}$ a $220 \mathrm{mg} / \mathrm{dL}$, lo que corresponde a $35 \mathrm{mg} / \mathrm{dL}$, es decir una diferencia de $16 \%$.

Por otra parte sabemos que:

$\mathrm{CV}_{\mathrm{B}}$ colesterol $=6 \%$ (dato obtenido de tablas de CVB).

$\mathrm{CV}_{\mathrm{A}}$ colesterol $=3 \%$ (dato real del laboratorio obtenido de los datos de imprecisión del control de calidad interno).

Si reemplazamos en la fórmula, la diferencia crítica o RCV (con un Z de 1,96) calculada es de $18,5 \%$ y la diferencia verdadera de $16 \%$; es decir el cambio de valores del colesterol no es significativo y se debe sólo a la variación biológica y analítica de este parámetro.

\section{Conclusiones y cierre del caso}

Las fuentes de variación entre dos exámenes seriados realizados en un mismo laboratorio, deben ser consideradas antes de decidir si hubo un cambio clínicamente significativo. El manejo del CVB y CVA para exámenes de uso habitual son necesarios para que los médicos puedan interpretar correctamente los exámenes de laboratorio de un paciente. Según el cálculo desarrollado en este artículo, el cambio de valores de colesterol del paciente "de la vida real" no es clínicamente significativo; sin embargo, el valor verdadero del colesterol oscila en éste alrededor de los $200 \mathrm{mg} /$
dL. Esto nos obligará a considerar otros factores de riesgo cardiovascular y a controlar a nuestro enfermo con un estudio de lípidos completo en forma periódica.

Especial cuidado se debe tener con analitos como marcadores tumorales o hemoglobina glicosilada que aumentan sus CVB en enfermedad. Es importante que los laboratorios pongan a disposición de sus médicos usuarios los CVA de sus técnicas, para que en los casos necesarios, junto con el dato del CVB, éstos puedan realizar los cálculos mostrados en este artículo. En este tema, al igual que en armonización, es importante que para el control de un paciente, el seguimiento se debe hacer en el mismo laboratorio, evitando variaciones adicionales.

\section{Referencias}

1. Guzmán AM, Solari S. [Traceability and harmonization in clinical laboratory]. Rev Med Chile 2009; 137: 713-5.

2. Fraser CG. Test result variation and the quality of evidence-based clinical guidelines. Clin Chim Acta 2004; 2; 346: 19-24.

3. Fraser CG. Inherent biological variation and reference values. Clin Chem Lab Med 2004; 42: 758-64.

4. Ricós C, Álvarez V, Cava F, García-Lario JV, Hernández A, Jiménez CV, et al. Current databases on biological variation: pros, cons and progress. Scand J Clin Lab Invest 1999; 59: 491-500.

5. Westgard QC. Biological Variation Database specifications. Disponible en: Westgard.com (Consultado el 28 de mayo de 2010).

6. Ricós C, Iglesias N, García-Lario JV, Simón M, Cava F, Hernández A, et al. Within-subject biological variation in disease: collated data and clinical consequences. Ann Clin Biochem 2007; 44: 343-52.

7. Statistical quality control for quantitative measurements procedures: Principles and definitions. Approved guideline (2006) C24-A3. Clinical and Laboratory Standards Institute.

8. Ricós C, Cava F, García-Lario JV, Hernández A, Iglesias $\mathrm{N}$, Jiménez CV, et al. The reference change value: a proposal to interpret laboratory reports in serial testing based on biological variation. Scand J Clin Lab Invest. 2004; 64: 175-84. 